\title{
The Influence of Turbulence on Characteristics of a Hybrid-Stabilized Argon-Water Electric Arc*
}

\author{
Jiř́ JENIŠTA**, Hidemasa TAKANA***, Hideya NISHIYAMA***, \\ Milada BARTLOVÁ****, Vladimír AUBRECHT**** and Petr KŘENEK** \\ ** Institute of Plasma Physics AS CR, v.v.i. \\ Za Slovankou 3, 18200 Praha 8, Czech Republic \\ E-mail: jenista@ipp.cas.cz \\ *** Institute of Fluid Science, Tohoku University \\ 2-1-1 Katahira, Aoba-ku, Sendai, Miyagi, 980-8577, Japan \\ **** Brno University of Technology \\ Technická 8, 61600 Brno, Czech Republic
}

\begin{abstract}
The paper presents numerical simulations of the discharge and near outlet regions of the hybrid-stabilized argon-water electric arc. Calculations were carried out for the assumption of laminar and turbulent plasma flow models, respectively. Results of calculations for currents 300-600 A show that the influence of turbulence is weak and the maximum difference for all the monitored physical quantities is less than $10 \%$. Comparison with available experiments exhibits good agreement.
\end{abstract}

Key words: Hybrid-Stabilized Electric Arc, Partial Characteristics, Mass Flow Rate, Large-Eddy Simulation, the Smagorinsky Sub-Grid Scale Model

\section{Introduction}

The so-called hybrid-stabilized electric arc, which was developed a decade ago at the Institute of Plasma Physics AS CR, v.v.i. (IPP AS CR, v.v.i.) in Prague, utilizes a combination of gas and vortex stabilization. (A picture and principle of it is shown in Figure 1). In the hybrid argon-water plasma torch, the arc chamber is divided into the short cathode part, where the arc is stabilized by tangential argon flow, and the longer part, which is stabilized by water vortex. This arrangement provides not only the additional stabilization of the cathode region and the protection of the cathode tip, but also offers the possibility of controlling plasma jet characteristics in a wider range than that of pure gas- or liquid-stabilized arcs. ${ }^{(1-2)}$ The arc is attached to the external water-cooled rotating disc anode at a few millimeters downstream of the torch orifice. The experiments made on this type of $\operatorname{torch}^{(1,3)}$ showed that the plasma mass flow rate, velocity and momentum flux in the jet can be controlled by changing the mass flow rate in the gas-stabilized section, whereas thermal characteristics are determined by the processes in the water-stabilized section.

The hybrid arc has been used at IPP AS CR, v.v.i., in the plasma spraying torch WSP ${ }^{\circledR} \mathrm{H}$ $(160 \mathrm{~kW})$ for spraying metallic or ceramic powders $\left(\mathrm{TiO}_{2}, \mathrm{Al}_{2} \mathrm{O}_{3}, \mathrm{ZrSiO}_{4}, \mathrm{~W}\right.$-based, Ni-based alloys, Al, steel) injected into the plasma jet. Some of the latest studies of physical and chemical processes in materials after their interaction with plasma can be found, e.g., in Refs. (4) (6). Several years ago, an experimental plasmachemical reactor PLASGAS equipped with the spraying torch $\mathrm{WSP}^{\circledR} \mathrm{H}$ was utilized for the innovative and environmentally friendly plasma treatment of waste streams with a view to their sustainable energetic and chemical valorization and to a reduction of the emission of greenhouse 
gases. ${ }^{(7-8)}$ Pyrolysis of biomass was experimentally studied in a reactor using crushed wood, sunflower seeds or rubber oil as model substances. A high content of a combustible mixture of hydrogen and carbon monoxide was produced (i.e., syngas). Production of higher hydrocarbons is suppressed due to higher temperatures and a high level of ultraviolet radiation in the reactor volume. Experiments with the PLASGAS reactor have currently been focused on the produced gas composition in dependence on the reactor atmosphere and jet characteristics for different conditions including inflow cold reaction gases as, e.g., oxygen, carbon dioxide and air.

Some of our previous calculations analyzed flow regimes, thermal, electrical characteristics and power losses from the hybrid-stabilized electric arc. ${ }^{(9-12)}$ Experiments carried out with the hybrid torch show the laminar structure of the plasma flowing out of the discharge chamber just behind the nozzle for currents up to $600 \mathrm{~A}$, i.e., the operational regime of our torch. Our previous calculation demonstrates that the Reynolds number based on the outlet diameter $6 \mathrm{~mm}$ reaches in the axial region 13,000 at maximum and decreases to 300 in arc fringes. The type of flow inside the discharge chamber is questionable since no diagnostic assessment is able to see inside the chamber and it is not clear if the laminar plasma stream is a result of the laminarization of the plasma flow at the outlet.

The aim of this research is to confirm through numerical simulation if plasma flow in our hybrid torch can be considered laminar or if turbulence should be considered. To check possible deviations from the laminar model, we utilized the Large-Eddy Simulation (LES) in the present model and compared our results against the laminar case. In our previous paper $^{(12)}$ we stated that the maximum detected discrepancy between the turbulent and laminar models is below $7 \%$ for the relative temperature difference at one point only at the arc axis downstream of the nozzle exit, but this time we studied turbulence in the discharge in greater detail. The domain for numerical calculation is shown in Figure 1 by a red-dashed line and includes the discharge area between the outlet nozzle for argon and the near-outlet region of the hybrid plasma torch.

Sections 2 and 3 provide information about the model assumptions, plasma properties, boundary conditions and the numerical scheme. Section 4 reveals the most important findings and a comparison of our calculated results with experiments.
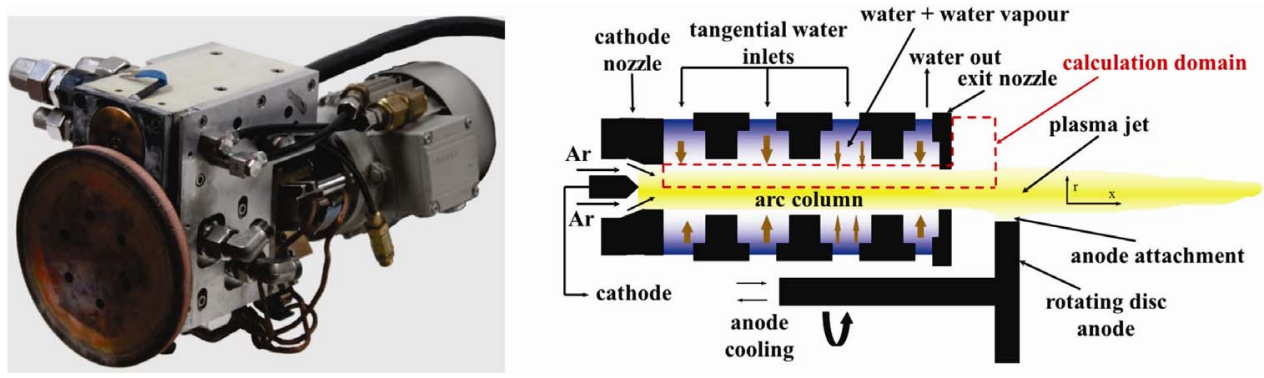

Fig. 1 The plasma spraying torch $\mathrm{WSP}^{\circledR} \mathrm{H}$ with hybrid stabilization (left), i.e., the combined stabilization of the arc by axial gas flow $\left(\mathrm{Ar}\right.$ or $\mathrm{N}_{2}$ ) and water vortex. The arc burns between the cathode, made of a small piece of zirconium pressed into a copper rod, and the water-cooled rotating disc anode, made of copper. The principle of the hybrid plasma torch WSP ${ }^{\circledR} \mathrm{H}$ with combined gas (Ar) and vortex (water) stabilizations is shown at right. Water is injected tangentially and creates a vortex in the chamber. The calculation domain is shown by a red-dashed line. 


\section{Physical Model}

The following assumptions for the model were applied:

1) The numerical model is two-dimensional with the discharge axis being the axis of symmetry;

2) Plasma flow is laminar/turbulent and compressible in the state of local thermodynamic equilibrium;

3) Argon and water create a uniform mixture in the arc chamber;

4) Only a self-generated magnetic field by the arc itself is considered;

5) Gravity effects are negligible;

6) The partial characteristics method for radiation losses from the arc is employed.

A couple of some comments on these assumptions:

1) The assumption of axial symmetry: The cylindrical discharge chamber (Figure 1) is divided into several sections by baffles with central holes. Water is injected tangentially into the chamber by three sets of three inlet holes (totaling 9 holes) placed equidistantly along the circumference at angles of $120^{\circ}$. The inner diameter of the water vortex is determined by the diameter of the holes in the baffles. Water is usually pumped under pressures of 0.39 MPa $(0.6 \mathrm{MPa})$ with flow rates of $10 \mathrm{l} / \mathrm{min}(16 \mathrm{l} / \mathrm{min})$. Higher pressures insure better hydrodynamic stability of the arc. Since water flows in a closed circuit, it is also exhausted at two positions along the arc chamber.

In order to facilitate seeing the plasma flow structure near the outlet, we included in our calculation domain the near-outlet region which extends up to $20 \mathrm{~mm}$ from the nozzle exit. In the experiment, the distance from the nozzle exit to the anode can be changed from 5 to $20 \mathrm{~mm}$. The regions close to the nozzle exit can be expected to remain undisturbed by the presence of the anode, while the more distant regions $(15-20 \mathrm{~mm})$ will be influenced by $3 \mathrm{D}$ effects (by the anode jet and anode processes), provided the anode is placed about $20 \mathrm{~mm}$ from the nozzle exit.

The result of considering that the two-dimensional assumption is valid in a major part of the domain is due to, a) cylindrical symmetry of the discharge chamber setup, b) tangential injection of water through the holes along the circumference, and c) the flexible distance between the nozzle exit and anode.

2) The assumption of a complete (uniform) mixing is a simplification of reality since, based on experiments, argon and water species do not mix homogeneously in the hybrid torch, especially at lower currents. This assumption was discussed in more detail in Ref. (9) and the conclusion was that this assumption can underestimate to some extent temperature and velocity in the axial discharge region.

The complete set of conservation equations representing the mass, electric charge, momentum and energy transport of such plasma can be written in the vector notation as follows:

continuity equation:

$\frac{\partial}{\partial t} \rho+\nabla \cdot(\rho \vec{u})=0$

momentum equations:

$\frac{\partial}{\partial t}(\rho \vec{u})+\nabla \cdot(\rho \vec{u} \vec{u})=-\nabla p+\nabla \cdot \vec{\tau}+\vec{j} \times \vec{B}, \tau_{i j}=\eta\left(\frac{\partial u_{i}}{\partial x_{j}}+\frac{\partial u_{j}}{\partial x_{i}}-\frac{2}{3} \delta_{i j} \frac{\partial u_{l}}{\partial x_{l}}\right)$, 
energy equation:

$\frac{\partial e}{\partial t}+\nabla \cdot[(e+p) \vec{u}-\lambda \nabla T]=\vec{j} \cdot \vec{E}+\Phi_{\text {diss }}-\dot{R} \quad$,

charge continuity equation:

$\nabla \cdot(\sigma \nabla \Phi)=0$

equation of state:

$p=\rho R_{g} T$

Here $\rho$ is the mass density, $\vec{u}$ the velocity vector containing the axial $(u)$ and radial (v) velocity components, $p$ the pressure, $\vec{\tau}$ the stress tensor, $\vec{j}$ the current density, $\vec{B}$ the magnetic field, $e$ the density of internal and kinetic energy produced or dissipated in the unit volume, $T$ the temperature, $\vec{E}$ the electric field strength, $\Phi_{\text {diss }}$ the viscous dissipation term, $\dot{R}$ the divergence of radiation flux, $\Phi$ the electric potential, $\lambda$ and $\sigma$ are the thermal and electrical conductivities, respectively, and $R_{g}$ is the specific gas constant.

The water-argon mixture can be described by the formula $\left(\mathrm{H}_{2} \mathrm{O}\right)_{(1-x)} \mathrm{Ar}_{\mathrm{x}}$ where the argon molar amounts " $\mathrm{x}$ " were selected from 0 to 1 with the step of 0.05 . The total number of 35 chemical species was considered ${ }^{(13)}$. For the temperature range $400-20000 \mathrm{~K}$, we presumed the following decomposition products: e, $\mathrm{H}, \mathrm{O}, \mathrm{Ar}, \mathrm{O}^{+}, \mathrm{O}^{2+}, \mathrm{O}^{3+}$, $\mathrm{O}^{-}, \mathrm{O}_{2}, \mathrm{O}_{2}^{+}, \mathrm{O}_{2}^{-}, \mathrm{O}_{3}, \mathrm{H}^{+}, \mathrm{H}^{-}, \mathrm{H}_{2}, \mathrm{H}_{2}^{+}, \mathrm{H}_{3}^{+}, \mathrm{OH}, \mathrm{OH}^{+}, \mathrm{OH}^{-}, \mathrm{HO}_{2}$, $\mathrm{HO}_{2}^{-}, \mathrm{H}_{2} \mathrm{O}, \mathrm{H}_{2} \mathrm{O}^{+}, \mathrm{H}_{3} \mathrm{O}^{+}, \mathrm{H}_{2} \mathrm{O}_{2}, \mathrm{Ar}^{+}, \mathrm{Ar}^{2+}, \mathrm{Ar}^{3+}$. The set of products is somewhat different for the temperature range $20-50,000 \mathrm{~K}$, including also multiple charged ions: e , $\mathrm{H}, \mathrm{O}, \mathrm{Ar}, \mathrm{O}^{+}, \mathrm{O}^{2+}, \mathrm{O}^{3+}, \mathrm{O}^{4+}, \mathrm{O}^{5+}, \mathrm{O}^{6+}, \mathrm{H}^{+}, \mathrm{Ar}^{+}, \mathrm{Ar}^{2+}$, $\mathrm{Ar}^{3+}, \mathrm{Ar}^{4+}, \mathrm{Ar}^{5+}, \mathrm{Ar}^{6+}$. The calculations were performed using the modified Newton method for the solution of the nonlinear equations system, which is composed of equations of Saha and mass action law type expressing individual complex components by means of basic ones (e, H, O, Ar). The system was completed by the usual particle and charge balance assuming quasineutrality and equilibrium.

The thermodynamic properties and the transport coefficients of this gas mixture were calculated according to the Chapman-Enskog method in the 4th approximation described, e.g., in ${ }^{(14)}$ for temperatures of $400-50,000 \mathrm{~K}^{(13)}$ and pressures of $0.1-0.3 \mathrm{MPa}$ in the local thermodynamic equilibrium.

Turbulence was modeled by Large-Eddy Simulation using the Smagorinsky sub-grid scale model $^{(15)}$ with the constant values of the Smagorinsky coefficient $\left(C_{S}=0.1\right)$ and the turbulent Prandtl number $\left(P r_{t}=0.9\right)$. To suppress turbulence near the walls, we applied a Van Driest damping function near the walls ${ }^{(15)}$ in the form

$$
l_{S}=C_{S} \Delta\left[1-\exp \left(-y^{+} / A^{+}\right)\right]
$$

where $l_{S}$ is the Smagorinsky lengthscale, $C_{S}$ is the Smagorinsky coefficient, $\Delta$ is the filter width, $y^{+}$is the distance from the wall normalized by the viscous lengthscale, and $A^{+}=25$. The eddy viscosity $\mu_{t}$ and the turbulent thermal conductivity $\lambda_{t}$ are modeled $\operatorname{as}^{(15)}$

$$
\mu_{t}=l_{S}^{2} \bar{S}=\left(C_{S} \Delta\right)^{2} \bar{S}
$$

$$
\lambda_{t}=c_{p} \mu_{t} / \operatorname{Pr}_{t}
$$


$\bar{S}$ is the characteristic filtered rate of strain and $c_{p}$ is the specific heat at constant pressure. The filter width $\Delta$ is proportional to the grid size with generally nonequidistant spacing $\Delta x_{i}$ and $\Delta y_{j}$, defined here as $\Delta \equiv \Delta_{k}=\sqrt{\Delta x_{i} \Delta y_{j}}$.

Radiation losses from the argon-water arc were calculated by the partial characteristics method for different molar fractions of argon and water plasma species as a function of temperature and pressure. The principle of the method is that the calculation of integrals over radiation frequencies in equations for radiation quantities is performed in advance to form functions Som and $\Delta$ Sim, called partial characteristics ${ }^{(16-17)}$. Partial characteristics include all time demanding integrations over frequencies. These functions are stored in data tables according to several parameters, such as plasma pressure, temperature and geometrical dimensions. The tables are then used in numerical simulations of plasma flow with spatial integrations only. The advantage of this method is that it directly calculates the reabsorption of radiation in the low-temperature regions of the arc.

The partial characteristics Som and $\Delta$ Sim are defined as follows: ${ }^{(18)}$

$$
\begin{aligned}
& \operatorname{Som}\left(T_{X}, T_{R},|R-X|\right)=\int_{0}^{\infty} B_{v}(X) \kappa_{v}(X) \times \exp \left(-\int_{X}^{R} \kappa_{v}(\eta) d \eta\right) d v, \\
& \Delta \operatorname{Sim}\left(T_{X}, T_{\xi}, \xi-X\right)=\int_{0}^{\infty}\left[B_{v}(X)-B_{v}(\xi)\right] \kappa_{v}(X) \kappa_{v}(\xi) \times \exp \left(-\int_{\xi}^{X} \kappa_{v}(\eta) d \eta\right) d v,
\end{aligned}
$$

where $B_{v}$ is the Planck function, $\kappa_{v}$ is the absorption coefficient, $T$ is the plasma temperature, $\xi, X$ are the radial coordinates and $R$ is the arc radius. For example, this method has been successfully used in some previous papers ${ }^{(19-20)}$ for the prediction of radiation intensities, radiation fluxes and the divergence of radiation fluxes for various temperature profiles of $\mathrm{SF}_{6}$ arc plasmas.

Continuous radiation due to photo-recombination and "bremsstrahlung" processes was included in the calculation as well as discrete radiation consisting of thousands of spectral lines. Broadening mechanisms of atomic and ionic spectral lines due to Doppler, resonance and Stark effects have been considered. The numbers of oxygen and argon lines included in the calculation for different species are shown in parenthesis: $\mathrm{O}(93), \mathrm{O}^{+}(296), \mathrm{O}^{2+}(190)$, $\mathrm{Ar}$ (739), $\mathrm{Ar}^{+}$(2781), $\mathrm{Ar}^{2+}$ (403), $\mathrm{Ar}^{3+}$ (73). In addition, molecular bands of $\mathrm{O}_{2}$ (Schuman-Runge system), $\mathrm{H}_{2}$ (Lyman and Verner systems), $\mathrm{OH}$ (transition $\mathrm{A}^{2} \Sigma^{+} \rightarrow \mathrm{X}^{2} \Pi_{\mathrm{i}}$ ) and $\mathrm{H}_{2} \mathrm{O}$ (several transitions) were also brought about. ${ }^{(21)}$ Absorption coefficient as a function of wavelength was calculated from infrared to far ultraviolet regions and the tables of partial characteristics for $1,000-35,000 \mathrm{~K}$.

\section{Boundary conditions and the numerical method}

The calculation region and the corresponding boundary conditions are presented in Figure 2. The dimensions are $3.3 \mathrm{~mm}$ for the radius of the discharge region, $20 \mathrm{~mm}$ for the radius of the outlet region and $78.32 \mathrm{~mm}$ for the total length. These dimensions agree with the hybrid torch experimental setup.

The dimension and shape of the outlet nozzle shown in Figure 2 correspond exactly to the experimental setup with one exception: the numerical model omits a small radius of the edges in order to decrease thermal erosion of the nozzle. The exact shape of the edges is our innovation. It is confirmed by our experiments that the nozzle undergoes additional erosion during the arc operation which is also not considered in the model. The higher the arc current, the higher is generally the erosion of the nozzle. The best choice for nozzle material is copper because of its high thermal conductivity; the water-cooled copper nozzle can transfer high heat fluxes from the plasma discharge. 
(a) Inlet boundary $(A B)$ is represented by the nozzle exit for argon. Along this boundary we assume the zero radial velocity component, $v=0$. Because of the lack of experimental data, the temperature profile $T(r, z=0)$ and the electric field strength $E_{z}=-\partial \Phi / \partial z=$ const. for a given current are calculated at this boundary. This is before the start of the fluid-dynamic calculation itself, iteratively from the Elenbaas-Heller equation including the radiation losses from the arc. (Our previous numerical experiments proved a weak dependence of the form of the boundary temperature profile on the overall solution). The inlet velocity profile $u(r)$ for argon plasma for the obtained temperature profile $T(r, z=0)$ is pre-calculated from the axial momentum equation under the assumption of a fully developed flow.

(b) Axis of symmetry $(B C)$. The zero radial velocity and symmetry conditions for the temperature, axial velocity and electric potential are specified here, i.e., $\partial T / \partial r=\partial u / \partial r=\partial \Phi / \partial r=0, v=0$.

(c) Arc gas outlet plane (CD). The zero electric potential $\Phi=0$ (the reference value) and zero axial derivatives of the temperature and velocity components are defined at CD, i.e., $\partial T / \partial z=\partial u / \partial z=\partial v / \partial z=0$.

(d) Arc gas outlet plane $(D E)$. The zero radial derivatives of the temperature, axial velocity, electric potential and zero radial velocity are defined here, $\partial T / \partial r=$ $\partial u / \partial r=\partial \Phi / \partial r=0, v=0$. Pressure is fixed at 1 atmosphere, $\mathrm{p}=1 \mathrm{~atm}$.

(e) Outlet wall and the nozzle (EF). We specify no slip conditions for velocities, $u=v=0$, constant values of $E_{r}$ and $E_{z}(\partial \Phi / \partial z=\partial \Phi / \partial r=0)$ and $T(r, z)=773 \mathrm{~K}\left(500^{\circ} \mathrm{C}\right)$ for the temperature of the nozzle.

(f) Water vapor boundary (FA). Along this line we specify the so-called "effective water vapor boundary," designated in Figure 2 as the "water vapor boundary" with a prescribed temperature of water vapor $T(R=3.3 \mathrm{~mm}, z)=773 \mathrm{~K}$. This is a numerical simplification of a more complex physical reality assumed to be near the phase transition water-vapor in the discharge chamber. The shape of the phase transition between water and vapor in the discharge chamber is not experimentally known and it is unclear as yet if the structure of the transition is simple or very complicated, for example, with a time-dependent form. Various irregularities in the transition, such as splitting the phase transition or water drops in the vapor phase, can increase complexity of the transition. In Ref. (22) the iteration procedure for determination of the mass flow rate $\dot{m}$ and the radius of the "water vapor boundary" for each current was proposed, based on comparing the available experimental temperature and velocity at the outlet and the electric potential drop in the chamber. As a result, it was concluded that the best fit between experiment and numerical simulation for all currents exists for a mean arc radius of $3.3 \mathrm{~mm}$. The corresponding values of water mass flow rates $\dot{m}$ are: $0.228 \mathrm{~g} \mathrm{~s}^{-1}(300 \mathrm{~A}), 0.286 \mathrm{~g} \mathrm{~s}^{-1}(350 \mathrm{~A}), 0.315 \mathrm{~g} \mathrm{~s}^{-1}(400 \mathrm{~A}), 0.329 \mathrm{~g} \mathrm{~s}^{-1}(500 \mathrm{~A})$, $0.363 \mathrm{~g} \mathrm{~s}^{-1}(600 \mathrm{~A})$. The magnitude of the radial inflow velocity is calculated from the definition of mass flow rate

$$
v(R)=\frac{\dot{m}}{2 \pi R \sum_{\Delta z} \rho(R, z) \Delta z}
$$

where, $\rho(R, z)$ is a function of pressure and thus dependent on the axial position $z$, $\Delta z$ being the distance between the neighboring grid points.

Due to practically zero current density in the cold vapor region (no current goes outside of the lateral domain edges), the radial component of the electric field strength is put at zero, i.e., $E_{r}=-\partial \Phi / \partial r=0$. The axial velocity component is set to zero, $u=0$.

Since we have not yet solved the equation for tangential velocity component $w$, distribution of $w$ in the discharge for the presented results was taken from our previous calculations. ${ }^{(23-24)}$ These were solved by the SIMPLER iteration procedure ${ }^{(25)}$ which enables 
calculation of $w$ for the axisymmetric case, i.e., $w$ is a function of $r$ and $z$ coordinates. The $w$ velocity acts here only through the centrifugal force $\rho w^{2} / r$ in the radial momentum equation. The influence of the tangential velocity component $w$ on the overall arc performance was studied in greater detail in our previous papers ${ }^{(23-24)}$ but it was found that its effect on the arc performance is small. In fact, the maximum difference in the outlet parameters was less than $2 \%$ regarding the case which excludes the tangential velocity component.

As the axial component of the current density $j_{z}$ in the discharge is much greater than the radial one $j_{r}$ we assume that the self-generated magnetic field by the arc itself has only the tangential component

$$
B_{\vartheta}(r, z)=\frac{\mu_{0}}{r} \int_{0}^{r} j_{z}(r, z) r d r,
$$

where $\mu_{0}$ is the vacuum permeability and $j_{z}(r, z)=-\sigma(r, z) \partial \Phi / \partial z$.

LU-SGS (Lower-Upper Symmetric Gauss-Seidel) algorithm ${ }^{(26-27)}$, belonging to the group of the density-based methods ${ }^{(28)}$ coupled with the Newtonian iteration method, are used for the integration of discretized Eqs. (1) (4) in time and space. To resolve compressible phenomena accurately, the Roe Flux Differential Scheme ${ }^{(29)}$ coupled with the third-order MUSCL-type (Monotone Upstream-centered Schemes for Conservation Laws) TVD (Total Variation Diminishing) scheme ${ }^{(30-31)}$ are used for a convective term. The electric potential from Eq. (4) is solved in a separate subroutine by the TDMA (Tri-Diagonal Matrix Algorithm) line-by-line method, including the block correction technique to speed up the convergence. Under-relaxation is employed to avoid divergence. From Eqs. (1) (4) we obtain $\rho, \rho \vec{u}, e$ and $\Phi$. Pressure is determined from the pressure- and temperature-dependence of the internal energy $U(p, T)=e(p, T)-0.5 \rho|\vec{u}|^{2}$. Temperature is calculated from the equation of state (5) $p / \rho=R_{g}(p, T) \cdot T$, using the pre-calculated values of the product $R_{g}(p, T) \cdot T$ as a function of temperature, pressure and argon molar fraction in the mixture. ${ }^{(13)}$

The computer program was written in the Fortran language. The task was solved on an oblique structured grid with nonequidistant spacing. The total number of grid points was 38,553, with 543 and 71 points in the axial and radial directions, respectively. Our numerical tests with different grid sizes confirmed the nearly grid-independent solutions for such a grid.

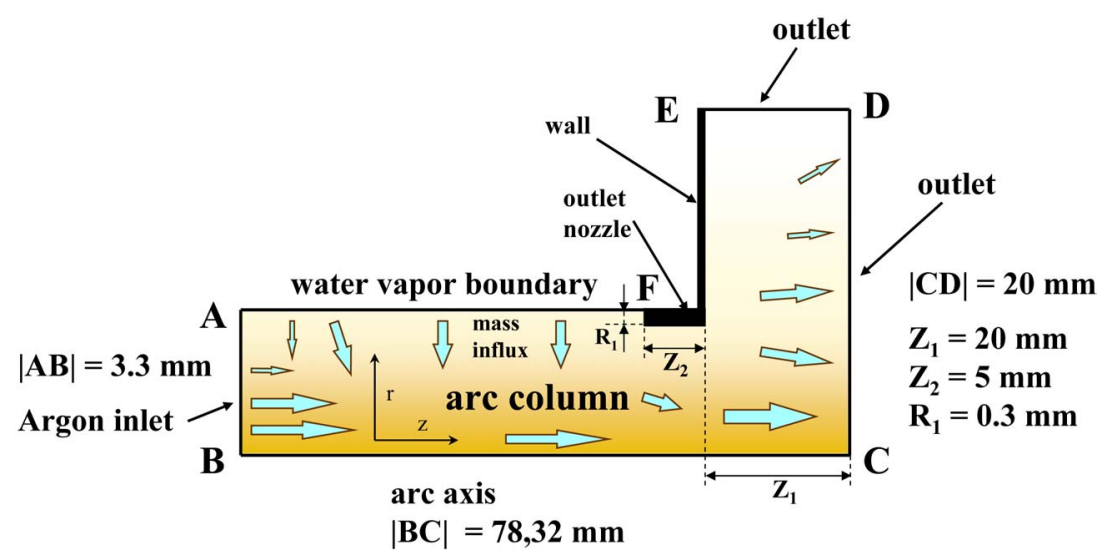

Fig. 2 Discharge area geometry. Inlet boundary $(\mathrm{AB})$ is represented by the nozzle exit for argon. The length of the discharge region is $78.32 \mathrm{~mm}$, the length of the outlet is $20 \mathrm{~mm}$. Adjacent to the line FE, the outlet nozzle and the wall of the hybrid plasma torch equipment is specified. 


\section{Results of calculation}

Calculations for turbulent and laminar models have been carried out for currents 300 , 400,500 and $600 \mathrm{~A}$. The mass flow rate for the water-stabilized section of the discharge was taken for each current between 300 and $600 \mathrm{~A}$ from our previously published work. ${ }^{(22)}$ The argon mass flow rate was varied in agreement with experiments in the interval from $22.5 \mathrm{slm}$ (standard liters per minute) to $40 \mathrm{slm}$, namely, 22.5, 27.5, 32.5 and $40 \mathrm{slm}$.

It was proved in experiments ${ }^{(32)}$ that part of argon is taken away before it reaches the torch exit because argon is mixed with vapor steam and removed through the water system of the torch. The amount of argon transferred in such a way from the discharge is around $50 \%$ for the currents studied. Since the mechanism of argon removal is not included in the model, we consider in the calculations that the argon mass flow rate present in the discharge equals one-half of argon mass flow rate at the torch inlet (i.e., for $22.5 \mathrm{slm}$ we take $50 \%$ of this amount, etc.).
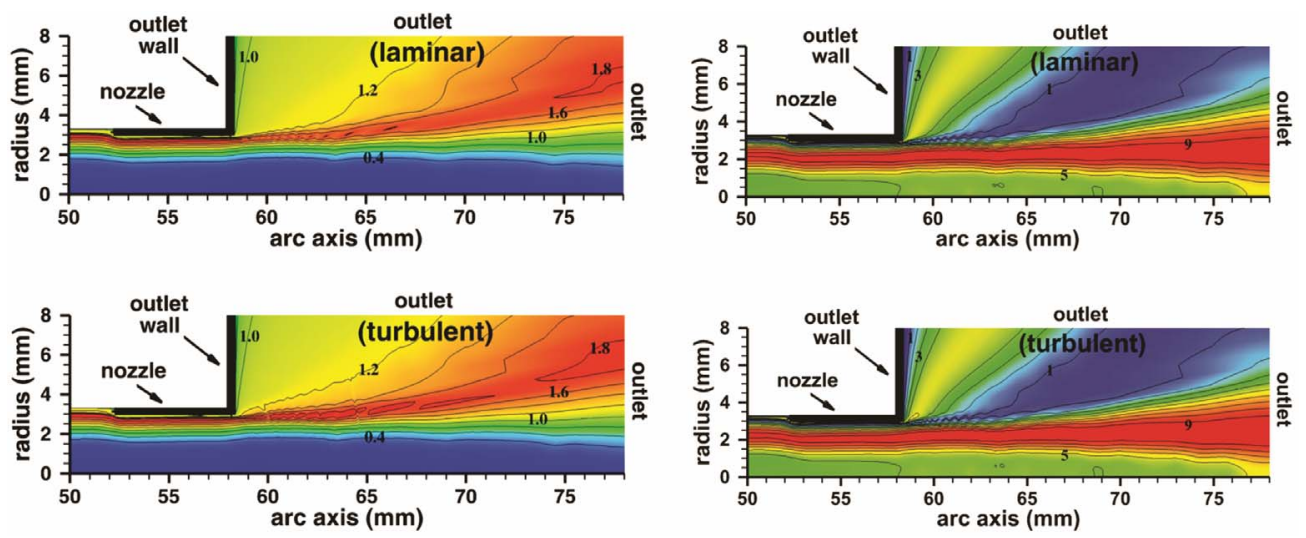

Fig. 3 Contours of the viscosity (left, $10^{4} \mathrm{~Pa} \cdot \mathrm{s}$ ) and thermal conductivity (right, $\mathrm{W} \cdot \mathrm{m}^{-1} \cdot \mathrm{K}^{-1}$ ) for $600 \mathrm{~A}$ and $40 \mathrm{slm}$ of argon for the laminar and turbulent models. Both viscosity and thermal conductivity are only mildly affected by turbulence.

Figure 3 displays the viscosity and thermal conductivity for $600 \mathrm{~A}$ and $40 \mathrm{slm}$ of argon under the assumptions of laminar and turbulent flow. Obviously, both properties are influenced only mildly by the turbulence. For viscosity, turbulent effects are stronger in small regions near the sharp edges of the outlet nozzle and also in the transition region between hot plasma and the surrounding atmosphere in the near-outlet region with high radial temperature and velocity gradients (shear layer). Thermal conductivity is affected only negligibly in the region with its maximum value (red color); the assumption of turbulent flow gives slightly higher values in this region.

Figure 4 shows velocity, temperature, pressure and the Mach number in the outlet nozzle and near-outlet regions for $600 \mathrm{~A}$ with a water mass flow rate of $0.363 \mathrm{~g} \mathrm{~s}^{-1}$ and 40 slm of argon. Among all the studied currents and argon mass flow rates, the results shown here demonstrate the largest variations of velocity, temperature, pressure and the Mach number just after the jet exhausts from the torch nozzle. Supersonic flow structure in the near-outlet region is obvious with clearly distinguished shock diamonds with the maximum Mach number being about 1.6 with $10,500 \mathrm{~m} \mathrm{~s}^{-1}$. The corresponding velocity and the Mach number maxima overlap with the temperature and pressure minima and vice versa. Since the pressure decreases at the torch exit to near atmospheric pressure, the computed contours correspond to an under-expanded atmospheric-pressure plasma jet. The turbulent case (b) provides somewhat lower values of all the presented physical quantities at the axial region 
of the arc.

(a) Laminar
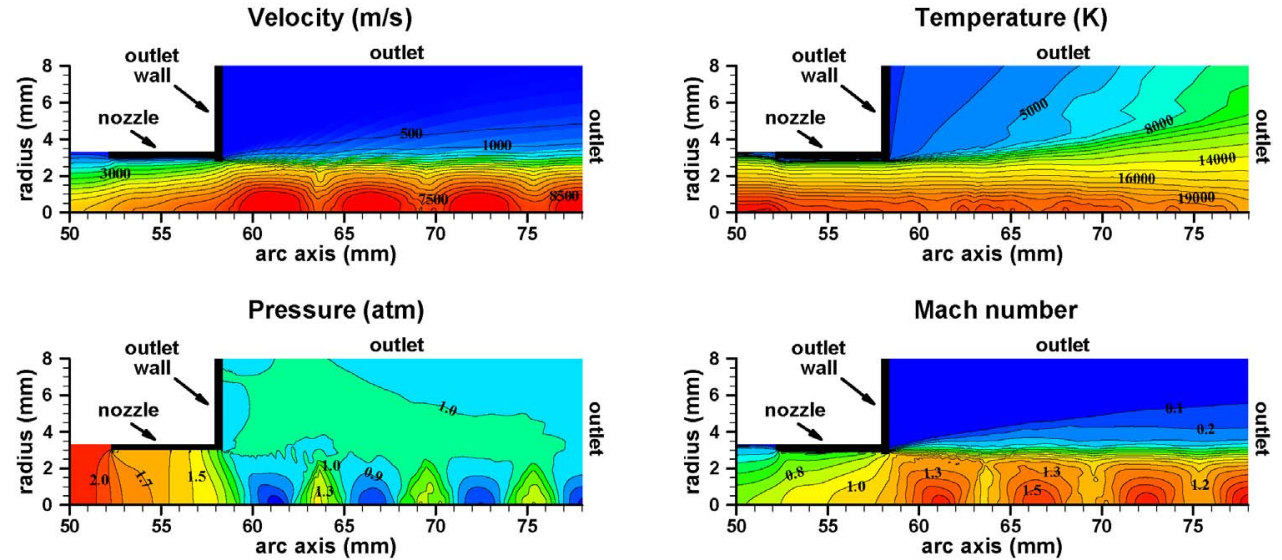

(b) Turbulent
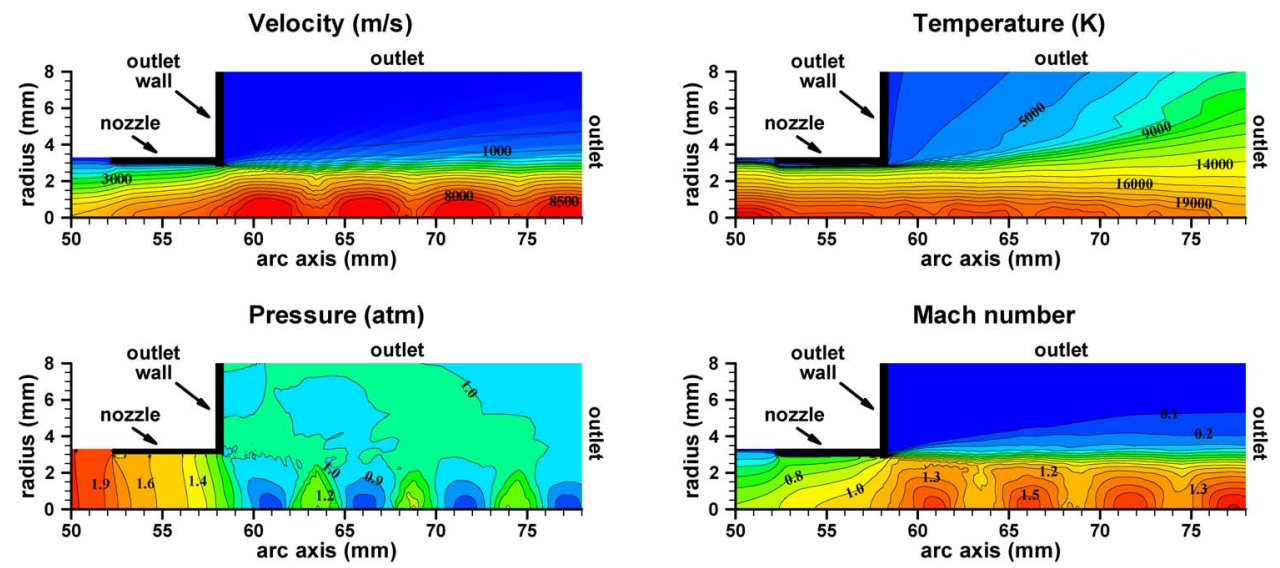

Fig. 4 Velocity, temperature, pressure and the Mach number contours in the outlet nozzle and near-discharge regions for the laminar (a) and turbulent (b) models. $600 \mathrm{~A}$, water mass flow rate is $0.363 \mathrm{~g} \mathrm{~s}^{-1}$, argon mass flow rate is $40 \mathrm{slm}$. Supersonic flow structure is obvious with clearly distinguished shock diamonds. The maximum Mach number reaches 1.5. Contour increments are $500 \mathrm{~m} \mathrm{~s}^{-1}$ for velocity, 1,000 K for temperature, $0.1 \mathrm{~atm}$ for pressure and 0.1 for the Mach number.

Figures 5 and 6 present the average relative difference between turbulent and laminar models within the volume of the discharge and of the near-outlet regions for temperature, velocity, pressure and the Mach and Reynolds numbers. The average relative difference for a given physical quantity $X$ is calculated as

$$
\Delta_{a v}^{X}=\frac{100}{N} \cdot \sum_{i=1}^{N} a b s\left(X_{\text {turb }}^{i}-X_{\text {lam }}^{i}\right) / X_{\text {turb }}^{i}
$$

where $N$ is the total number of grid points in the domain, represented by the discharge volume and the volume of the region downstream of the nozzle exit, $2 \mathrm{~mm}$ long and $6.6 \mathrm{~mm}$ thick (3.3 mm is the radius); "turb" and "lam" stand for the turbulent and laminar values of $X$, respectively.

One can see that the lowest difference occurs for temperature $(\sim 2.5 \%)$, a bit higher occur for velocity, pressure and the Mach number (below 5\%) and the highest difference 
occurs for the Reynolds number (up to 10\%). The sizes of the dots in the plots (i.e., the turbulence) increase with current for all displayed quantities.
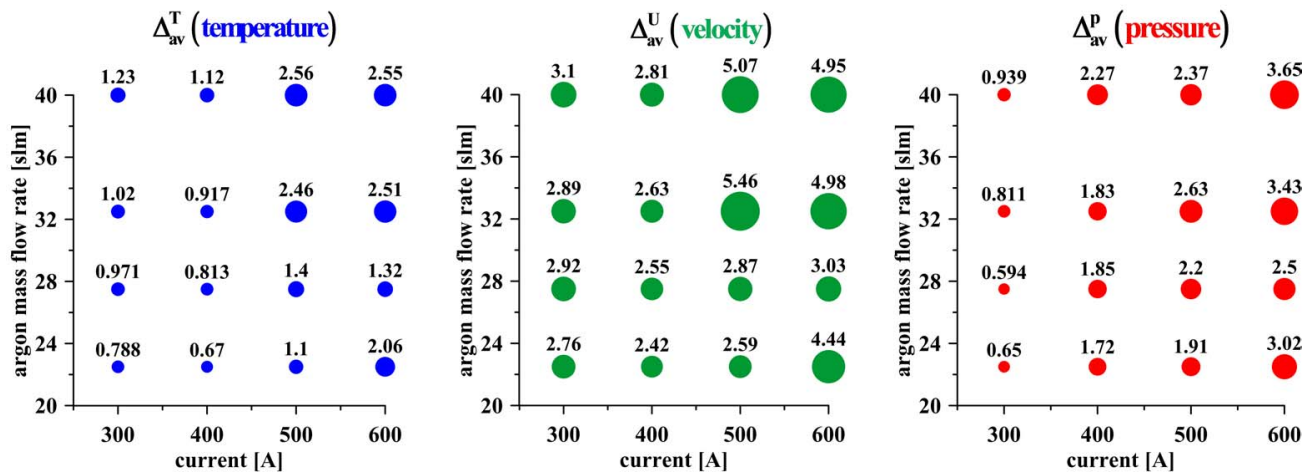

Fig. 5 The average relative difference in per cent between turbulent and laminar models within the volume of the discharge and the region downstream of the nozzle exit for temperature, velocity and pressure.
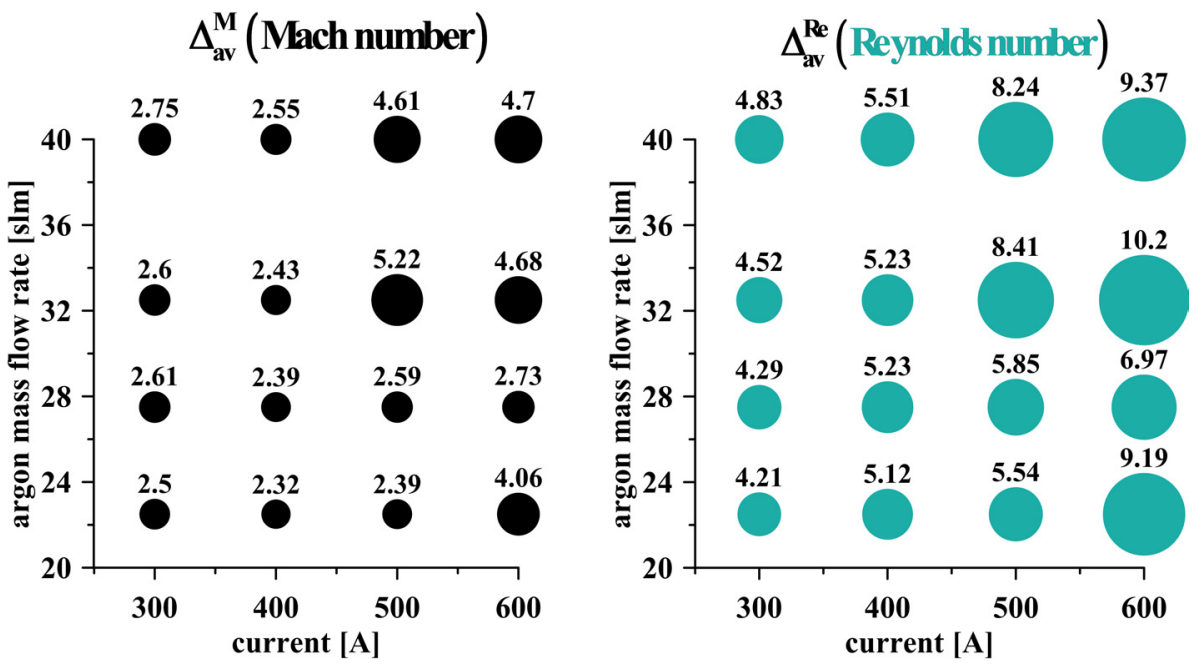

Fig. 6 Identical to Fig. 5 except for the Mach and Reynolds numbers.
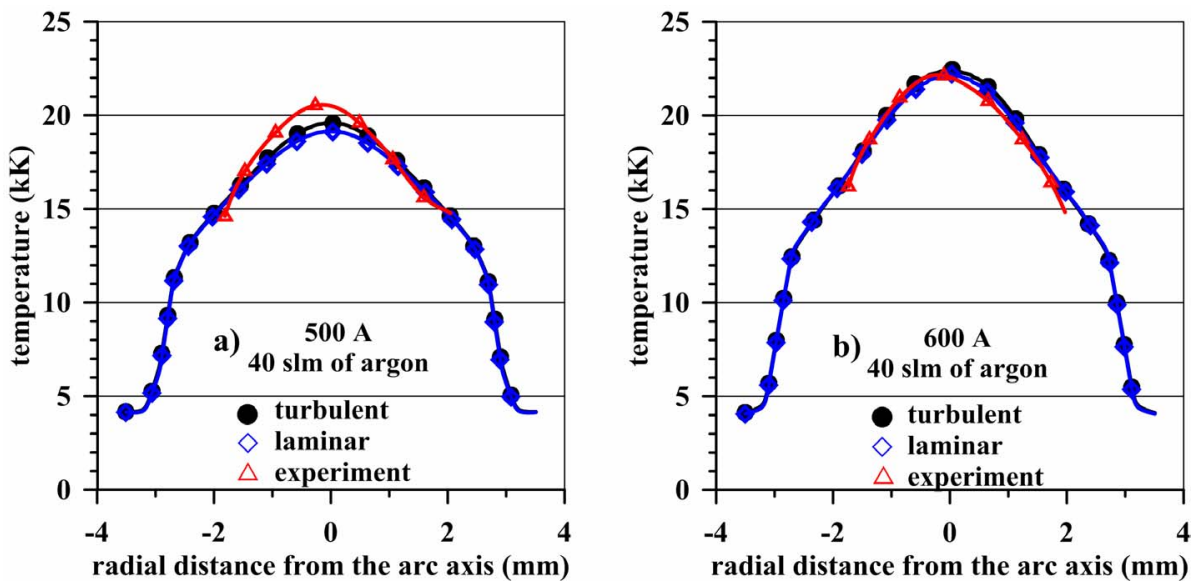

Fig. 7 Experimental and calculated radial temperature profiles $2 \mathrm{~mm}$ downstream of the nozzle exit for (a) $500 \mathrm{~A}$ and (b) $600 \mathrm{~A}$ at 40 slm of argon. 
Figure 7 compares measured and calculated radial temperature profiles $2 \mathrm{~mm}$ downstream of the nozzle exit for $500 \mathrm{~A}$ and $600 \mathrm{~A}$ for $40 \mathrm{slm}$ of argon. The temperature profiles for the laminar (blue line) and turbulent (black line) models nearly overlap. Very good agreement is demonstrated between experiment and calculation for $500 \mathrm{~A}$, and excellent agreement for $600 \mathrm{~A}$ where the measured profile nearly coincides with our calculation.

In our experiment, ${ }^{(33)}$ the radial profiles of the temperature near the nozzle exit were calculated from optical emission spectroscopy measurements. The procedure is based on the ratio of emission coefficients of the hydrogen $\mathrm{H}_{\beta}$ line and four argon ionic lines using calculated Local Thermodynamic Equilibrium (LTE) composition of the plasma for various argon mole fractions as a function of temperature. ${ }^{(13)}$ From the calculated molar fractions of hydrogen and argon, it is easy to obtain emission coefficients of $\mathrm{H}_{\beta}$ and argon lines. The temperature corresponding to an experimental ratio of emission coefficients is then found by cubic spline interpolation on the theoretical data.

\section{Conclusions}

The results obtained for currents 300-600 A and for argon mass flow rates 22.5-40 slm can be summarized as follows:

(a) Turbulence is not a significant phenomenon in the discharge and near-outlet regions of the hybrid-stabilized argon-water electric arc:

- Maximum relative difference of each of the monitored physical quantities calculated at the arc axis, along the radius near the outlet nozzle and within the volume of the discharge and near-outlet region, reaches less than $10 \%$.

- Difference generally increases with current; the maximum value of the difference occurs for the Reynolds number. The maximum value of the Reynolds number is $\sim 13,000$.

(b) Turbulent effects are stronger in small regions near sharp edges of the outlet nozzle and in the transition region between hot plasma and the surrounding atmosphere in the near-outlet region with high radial temperature and velocity gradients (shear layer). From our numerical simulation, we found that heat transfer from the arc to the nozzle with and without consideration of the turbulence is nearly the same because differences in temperature and velocity fields in the vicinity of the nozzle are negligible.

(c) Comparison with available experimental data demonstrates very good agreement for radial temperature profiles.

\section{Acknowledgments}

The authors are grateful for the financial support under the Fluid Science International COE Program of the Institute of Fluid Science, Tohoku University, Sendai, Japan, and for their computer facilities. Financial support from the Grant Agency of the Czech Republic (GA CR) 205/11/2070 is also gratefully acknowledged. The access to computing and storage facilities owned by the parties and projects contributing to the National Grid Infrastructure MetaCentrum, provided under the program "Projects of Large Infrastructure for Research, Development, and Innovations" (LM2010005), is likewise highly appreciated.

\section{References}

(1) Březina, V., Hrabovský, M., Konrád, M., Kopecký, V. and Sember, V., New plasma spraying torch with combined gas-liquid stabilization of arc, Proc. 15th Int. Symp. on Plasma Chemistry (ISPC 15, 2001), pp. 1021-26.

(2) Hrabovský, M., Kopecký, V. and Sember, V., Effect of Gas Properties on 
Characteristics of Hybrid Gas/Water Plasma Spraying Torch, Proc. 16th Int. Symp. on Plasma Chemistry (ISPC 16, 2003), on CD-ROM.

(3) Hrabovský, M., Kopecký, V., Sember, V., Kavka, T., Chumak, O. and Konrád, M., Properties of hybrid water/gas DC arc plasma torch, IEEE Trans. on Plasma Sci., Vol. 34 (2006), pp. 1566-75.

(4) Chráska, T., Neufuss, K., Dubský, J., Ctibor, P. and Rohan, P., Fabrication of bulk nanocrystalline alumina-zirconia materials, Ceramics International, Vol. 34 (2008), pp. 1229-36.

(5) Chráska, T., Neufuss, K., Dubský, J., Ctibor, P. and Klementová, M., Fabrication of Bulk Nanocrystalline Ceramic Materials, J. Thermal Spray Technology, Vol. 17 (2008), pp. 872-77.

(6) Ctibor, P., Ageorges, H., Sedláček, J. and Čtvrtlík, R., Structure and properties of plasma sprayed BaTiO3 coatings, Ceramics International, Vol. 36 (2010), pp. 2155-62.

(7) Van Oost, G., Hrabovský, M., Kopecký, V., Konrád, M., Hlína, M., Kavka, T., Chumak, O., Beeckman, E. and Verstraeten, J., Pyrolysis of waste using a hybrid argon-water stabilized torch, Vacuum, Vol. 80 (2006), pp. 1132-37.

(8) Van Oost, G., Hrabovský, M., Kopecký, V., Konrád, M., Hlína, M. and Kavka, T., Pyrolysis/gasification of biomass for synthetic fuel production using a hybrid gas-water stabilized plasma torch, Vacuum, Vol. 83 (2008), pp. 209-12.

(9) Jeništa, J., Numerical modeling of hybrid stabilized electric arc with uniform mixing of gases, IEEE Trans. on Plasma Sci., Vol. 32 (2004), pp. 464-72.

(10) Jeništa, J., Bartlová, M. and Aubrecht, V., Numerical modeling of electric arcs with water wartex and hybrid stabilizations, Complex Systems, AIP Conf. Proceedings 982 (2007), pp. 554-60.

(11) Jeništa, J., Takana, H., Nishiyama, H., Bartlova, M., Aubrecht, V. and Hrabovský, M., Parametric study of hybrid argon-water stabilized arc under subsonic and supersonic regimes, J. of High Temperature Material Processes, Vol. 14 (2010), pp. 63-76.

(12) Jeništa, J., Takana, H., Nishiyama, H., Bartlová, M., Aubrecht, V., Křenek, P., Hrabovský, M., Kavka, T., Sember, V. and Mašláni A., Integrated parametric study of a hybrid-stabilized argon-water arc under subsonic, transonic and supersonic plasma flow regimes, J. Phys. D: Appl. Phys., Vol. 44 (2011), 435204, 20pp.

(13) Křenek, P., Thermophysical properties of $\mathrm{H}_{2} \mathrm{O}-\mathrm{Ar}$ plasmas at temperatures 400-50,000 K and pressure 0.1 Mpa, Plasma Chem. and Plasma Processes, Vol. 28 (2008), pp. 107-22.

(14) Křenek, P. and Něnička, V., Calculation of transport coefficients of a gas mixture in the 4th approximation of Enskog-Chapman theory, Acta Technica CSAV, Vol. 28 (1984), pp. 420-33.

(15) Pope, S. B., Turbulent Flows, (2000), Cambridge University Press.

(16) Soloukhin, R. I., Radiative Heat Transfer in High-Temperature Gases, (1980), London: Hemisphere.

(17) Sevast'yanenko, V. G., Radiation transfer in a real spectrum. Integration over frequency, J. Eng. Phys., Vol. 36 (1979), pp. 138-48.

(18) Aubrecht, V., Bartlová, M., Urban, F. and Valenta, J., Partial characteristics of radiation for thermal plasmas in $\mathrm{H} 2 \mathrm{O}$ and argon, Proc. 15th Int. Conf. on Gas Discharges and their Applications (GD 2004), pp. 141 - 44.

(19) Aubrecht, V. and Lowke, J., J., Calculations of radiation transfer in SF6 plasmas using the method of partial characteristics, J. Phys. D: Appl. Phys., Vol. 27 (1994), pp. 2066-73.

(20) Aubrecht, V. and Bartlová, M., Calculations of radiative heat transfer in SF6 arc 
plasmas, IEEE Trans. on Plasma Sci., Vol. 25 (1997), pp. 815-23.

(21) Bartlová, M. and Aubrecht, V., Photoabsorption of diatomic molecules, Czech. J. Phys., 56 Suppl. B (2006), pp. B632-37.

(22) Jeništa, J., Water-vortex stabilized electric arc: III. Radial energy transport, determination of water-vapour-boundary and arc performance, J. Phys. D: Appl. Phys., Vol. 36 (2003), pp. 2995-3006.

(23) Jeništa, J., Water-vortex stabilized electric arc: I. Numerical model, J. Phys. D: Appl. Phys., Vol. 32 (1999), pp. 2763-76.

(24) Jeništa, J., Kopecký, V. and Hrabovský, M., Effect of vortex motion of stabilizing liquid wall on properties of arc in water plasma torch, Annals of the New York Academy of Sciences, vol 891 (1999), pp. 64-71.

(25) Patankar, S. V., Numerical Heat Transfer and Fluid Flow, (1980), New York: McGraw-Hill.

(26) Jameson, A. and Yoon, S., Lower-upper implicit schemes with multiple grids for the Euler equations, AIAA J., Vol. 25 (1987), pp. 929-35.

(27) Yoon, S. and Jameson, A., Lower-upper symmetric-Gauss-Seidel method for the Euler and Navier-Stokes equations, AIAA J., Vol. 26 (1988), pp. 1025-26.

(28) Kwak, D., Kiris, C. and Housman, J., Implicit methods for viscous incompressible flows, Computers \& Fluids, Vol. 41 (2011), pp. 51-64.

(29) Roe, P. L., Approximate Riemann solvers, parameter vectors, and difference schemes, J. Comput. Phys., Vol. 43 (1981), pp. 357-72.

(30) Leer, van B., Towards the ultimate conservative difference scheme: V. A second-order sequel to Godunov's method, J. Comput. Phys., Vol. 32 (1979), pp. 101-36.

(31) Chung, T. J., Computational Fluid Dynamics, (2010), New York: Cambridge University Press, 2nd edn.

(32) Kavka, T., Chumak, O., Sember, V. and Hrabovský, M., Processes in Gerdien arc generated by hybrid gas-water torch, Proc. 28th Int. Conf. on Phenomena in Ionized Gases (ICPIG 2007), pp. 1819-22.

(33) Sember, V. and Maslani, A., A Simple spectroscopic method for determining the temperature in $\mathrm{H}_{2} \mathrm{O}-\mathrm{Ar}$ thermal plasma jet, J. of High Temperature Material Processes, Vol. 13 (2009), pp. 217-28. 